\title{
Does Liquid-Liquid Phase Separation Drive Peptide
}

\section{Folding?}

Dean N. Edun, Meredith R. Flanagan, Arnaldo L. Serrano*

Department of Chemistry and Biochemistry, University of Notre Dame, Notre Dame, Indiana 46556, United States

\section{Corresponding Author}

*arnaldo.serrano@nd.edu

\begin{abstract}
Proline-arginine (PR) dipeptide repeats have been shown to undergo liquid-liquid phase separation and are an example of a growing number of intrinsically disordered proteins that can assemble into membraneless organelles. These structures have been posited as a nucleation site for pathogenic protein aggregation. As such, a better understanding of the effects that the increased local concentration and volumetric crowding within droplets has on peptide secondary structure is necessary. Herein we use Fourier transform infrared (FTIR) and two-dimensional infrared (2DIR) spectroscopy to show that formation of droplets by PR20 accompany changes in the Amide-I spectra consistent with folding into poly-proline helical structures.
\end{abstract}

KEYWORDS Liquid-Liquid Phase Separation, Droplets, Secondary Structure, Folding, 2DIR 
Roughly $44 \%$ of human proteins contain disordered segments of $>30$ amino acids. ${ }^{1}$ As protein folding often buries hydrophobic segments prone to self-association, some intrinsically disordered proteins and peptides (IDPs) susceptible to aggregation., ${ }^{2,3}$ More recently, many IDPs have been found to spontaneously organize into phase separated liquid droplets through coacervation. ${ }^{1,4,5}$ While liquid-liquid phase separation (LLPS) is often driven by charge-charge interactions, ${ }^{6,7}$ weak intermolecular interactions, such as pi stacking ${ }^{8,9}$ or volumetric crowding, ${ }^{7,10,11}$ can also provide the necessary driving force. This results in a peptide assembly mechanism that is distinct from amyloid formation. Functional forms of LLPS are responsible for producing membraneless organelles, especially within the nucleus of the cell. ${ }^{8,12}$ Aberrant LLPS has been proposed as a cause for cellular dysfunction. ${ }^{13}$ Upon droplet formation, local protein concentrations can increase by a factor of $50 .{ }^{14}$ As protein aggregation into potentially toxic amyloids is a highly nonlinear kinetic process, such an increase in local concentration could have a drastic effect on the stability of soluble protein. ${ }^{15,16}$ To begin to address this question, a better understanding of the effects that the increased local concentration and volumetric crowding within droplets has on peptide secondary structure is necessary. As such, a more elementary question of interest is whether liquid droplets promote protein folding of any kind for the otherwise unstructured IDPs. As high polymer volume fractions are known to stabilize protein folds, ${ }^{17}$ measurable secondary structure formation may accompany LLPS. Herein we present an approach to determine whether droplet formation promotes secondary structure folding for a model IDP using Fourier transform infrared (FTIR) and two-dimensional infrared (2DIR) spectroscopy.

Prior work on structure determination within droplets have been limited to circular dichroism (CD) spectroscopy and nuclear magnetic resonance (NMR) spectroscopy on gravimetrically isolated bulk phases. ${ }^{18}$ However, experiments where the phases no longer interact may not exhibit 
the same dynamics and structural changes that occur when both phases are present, due to the absence of an interface. In this respect 2DIR has an advantage. For example, CD measurements of protein secondary structure are optimized for optical densities (OD) below $1,{ }^{19}$ while the turbidity of a solution of LLPS droplets can easily reach a $1 \mathrm{~mm} \mathrm{OD}_{250}$ of greater than 1 . 2DIR spectroscopy however, when implemented using a 4-frame phase cycling pulse sequence, can eliminate the largest scattering artifacts, allowing us to directly measure LLPS droplets in situ. ${ }^{20,21}$ Similarly, many powerful NMR structural techniques rely on fast rotational correlation times, a limitation that 2DIR does not share.
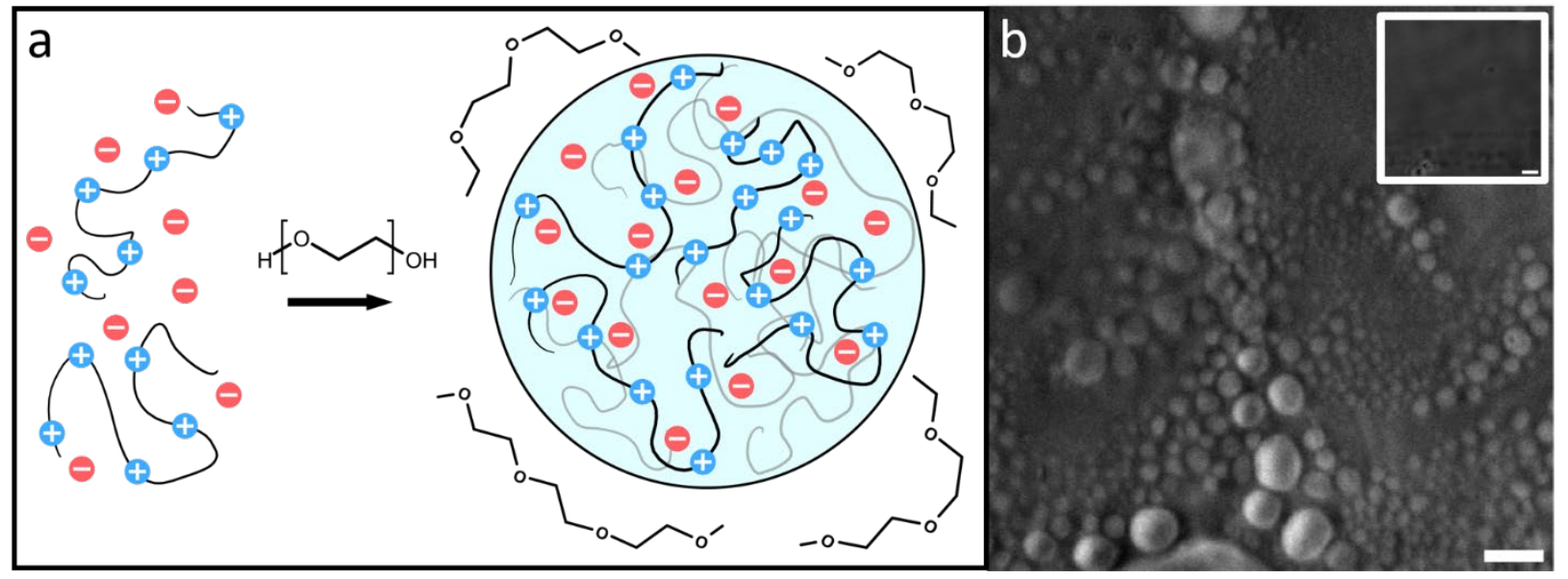

Figure 1. a) A cartoon of flexible protein segments undergoing phase separation through volumetric exclusion induced by a crowding agent, Polyethylene Glycol (PEG). b) PR20 phase separated droplets can be seen after the addition of PEG 300 using phase contrast microscopy. Inset shows a $\mathrm{PR}_{20}$ sample before addition of PEG 300. Scale bars represent $10 \mu \mathrm{m}$.

We chose, as a model system, the dipeptide repeat (DPR) $\mathrm{PR}_{20}$ (i.e. twenty repeats of prolinearginine). Our choice of this model system is partially motivated by the role of PR DRPs in Amyotrophic Lateral Sclerosis (ALS), where the length of the PR repeat correlates with 
cytotoxicity. ${ }^{25}$ Boeynaems and coworkers have shown that PR DPRs can phase separate with RNA. ${ }^{22}$ Past studies have also shown that PR DPRs co-localize with proteins and RNAs into liquid droplets in the nucleus of cells. ${ }^{23,24}$ Furthermore, PR repeats can undergo phase separation without a polyanionic polymer in buffered solution in the presence of $30 \% \mathrm{v} / \mathrm{v}$ polyethylene glycol (PEG). ${ }^{22} \mathrm{PEG}$ acts as a molecular crowder, reducing the intermolecular interaction strength needed to drive phase separation. ${ }^{11}$ The droplets, shown in cartoon form in Figure 1a, are likely to be composed solely of $\mathrm{PR}_{20}$, water, and buffer ions while PEG supplies the necessary depletion force for the droplets to form. ${ }^{10,26-28}$ Figure $\mathbf{1 b}$ shows droplets formed by $\mathrm{PR}_{20}$ in the presence of PEG using phase contrast microscopy.

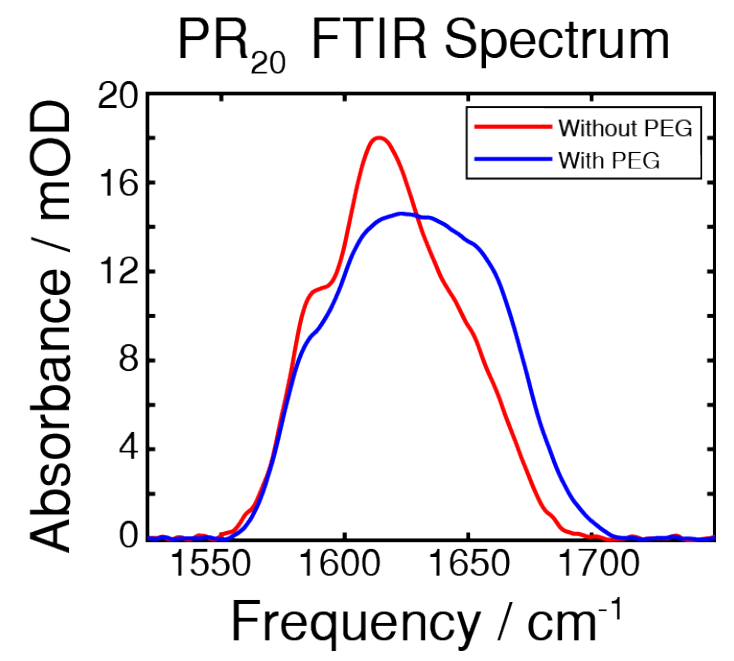

Figure 2. The FTIR spectra of the $\mathrm{PR}_{20}$ peptide taken in deuterated potassium phosphate buffer, with and without $30 \%$ PEG, as indicated. Peaks at 1585 and $1610 \mathrm{~cm}^{-1}$ comprise the arginine sidechains while a peak near $1650 \mathrm{~cm}^{-1}$ originates from the Amide-I stretch of randomly coiled peptide. After adding PEG, the spectrum broadens and shifts. 
An FTIR spectrum of the peptide in buffer shows peaks at around 1585 and $1610 \mathrm{~cm}^{-1}$ corresponding to the near-degenerate vibrational modes of the arginine sidechain $\mathrm{C}=\mathrm{N}$ stretches (Figure 2). ${ }^{29}$ A shoulder at around $1650 \mathrm{~cm}^{-1}$ corresponds to random-coil Amide-I. ${ }^{30}$ The FTIR spectrum after adding PEG shows a broadening of the absorption throughout the 1620 to $1650 \mathrm{~cm}^{-1}$ region.

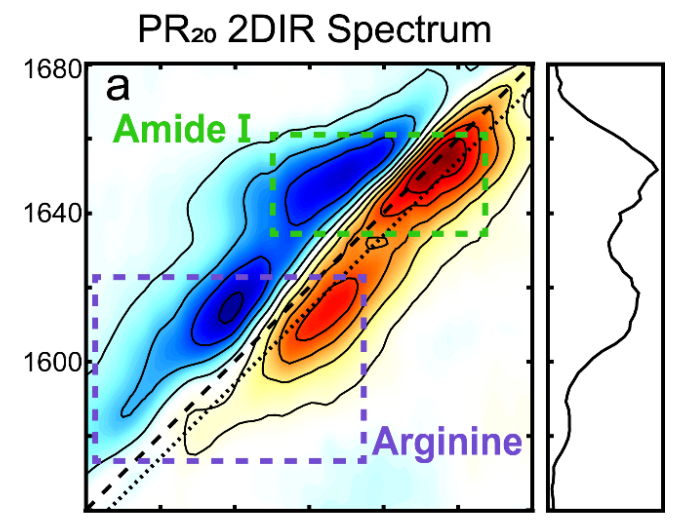

$\mathrm{PR}_{20}$ with $30 \%$ PEG 2DIR Spectrum
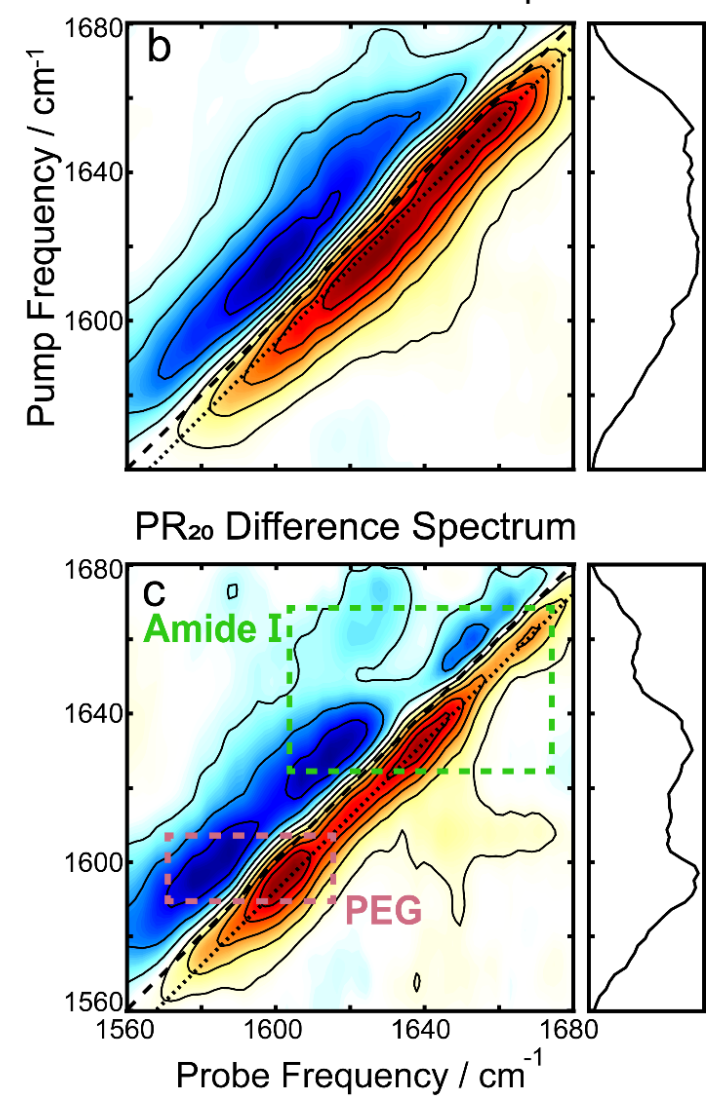
Figure 3. a) The 2DIR spectrum of $\mathrm{PR}_{20}$ without PEG shows a prominent peak corresponding to random-coils as well as the two arginine peaks. b) After adding PEG, the spectrum broadens along the diagonal. c) The difference spectrum of the first two spectra. The large peak at $1600 \mathrm{~cm}^{-1}$ originates from PEG. The two peaks at pump frequencies 1630 and $1665 \mathrm{~cm}^{-1}$ of the amide I region matches the peak locations for polyproline helices. ${ }^{31,32}$ For all spectra, the dotted line indicates where the diagonal cuts (panels to the right) are taken.

To further resolve features in the FTIR spectrum, we collected 2DIR spectra. The 2D spectrum of the $\mathrm{PR}_{20}$ before the addition of PEG (Figure 3a) shows a set of distinct peak pairs. Every FTIR transition corresponds to a red and blue peak pair in the 2D spectrum (red for ground state bleach/stimulated emission and blue for excited state absorption). Upon addition of PEG (Figure 3b) the spectrum broadens along the diagonal. A difference spectrum can be used to separate the contributions of the free peptide from the peptide sequestered into droplets, ${ }^{33}$ which is shown in Figures 3c. In the difference spectrum, a prominent new peak pair appears at pump frequency $1600 \mathrm{~cm}^{-1}$ which originates from PEG itself (see Figure S1 in the supporting information), as well as two new peak pairs at pump frequencies of 1630 and $1665 \mathrm{~cm}^{-1}$. There are no prominent blue peaks along the diagonal of the difference spectrum which one would expect from subtracting the random coil signatures in Figure 3a. This can occur if the new signatures in the Figure 3b were significantly larger, per molecule, than those in Figure 3a.

The previous observation can best be explained if the transition dipole moments, $\mu_{10}$, for the new signatures are larger than those of random coil transitions, as 2DIR signals have a $\mu_{10}^{4}$ dependence. This is often the case for peptide secondary structures, where coupling of alighted backbone modes form extended vibrational excitons. ${ }^{34,35}$ The near even split of the two new transitions, 1630 and $1665 \mathrm{~cm}^{-1}$, around the random coil frequency, $1650 \mathrm{~cm}^{-1}$, might suggest that 
these transitions arise from exciton splitting within a single structure. A possible candidate for such a species would be an extended beta sheet structure. ${ }^{30,36}$ We believe this is unlikely to be the case for this peptide, as the alternating prolines reduce the number of possible H-bonds across strands of the sheet. An alternative explanation is that the two new peaks correspond to two new species that are more favorably formed in the presence of prolines. In the following, we examine whether the peaks at 1630 and $1665 \mathrm{~cm}^{-1}$ can be explained by polyproline helices.

The two varieties of polyproline helical structures are polyproline I (PPI) and polyproline II (PPII). Both are left-handed with no hydrogen bonding along the backbone. The PPII helix is long and extended with trans peptide bonds whereas the rarer PPI helix is compacted with cis peptide bonds which are allowed by the conformational strain imparted by the proline residue. ${ }^{37,38}$ Past FTIR studies for the structures demonstrate low frequency peaks at $1628 \mathrm{~cm}^{-1}$ for PPI and 1637 $\mathrm{cm}^{-1}$ for PPII, with PPII also having a high frequency peak around $1660 \mathrm{~cm}^{-1} \cdot 31,32$ Comparing to our 2DIR spectra, this suggests $\mathrm{PR}_{20}$ may take on a mixture of PPI and PPII structure within the droplet phase.

We simulated 2D spectra of both polyproline structures to compare with experiment. The simulations were performed using a transition dipole coupling, floating oscillator model on idealized poly-proline structures, using homogenous line-widths and diagonal disorders typical of hydrated peptides (see supporting information for details). ${ }^{39}$ A comparison between the experimental 2DIR difference spectrum and the simulations can be found in Figure 4. The bottom panel shows the one to one sum of the calculated 2DIR spectra of PPI and PPII (individual spectra can be found in Figure S2). The peak positions (1637 and $\left.1659 \mathrm{~cm}^{-1}\right)$ and lineshapes in the simulation agree well, overall, with the amide-I region of the experimental spectrum, considering the simplicity of the model. A potential complication in interpretation of the spectra are past 
observations that intramolecular backbone hydrogen bonds can redshift the proline amide-I stretch by $25 \mathrm{~cm}^{-1} \cdot{ }^{40}$ Such hydrogen bonds are not present in the polyproline structures simulated here, and yet we observe a good match with experiments, suggesting that this phenomenon is unlikely to be occurring in the droplets. To determine the intensity of the polyproline peaks relative to random coil, we also simulated the 2D spectrum of a nine residue random coil (not shown). The intensities of the PPI and PPII peaks were, respectively, about 3.5 and 2 times more intense than that of the random coil peak. This is consistent with our observation that the new features upon droplet formation dominate the 2DIR difference spectra. In addition, the intensities from the simulations were used to estimate the relative population of the two species using the peak intensities in the experiment, yielding a ratio of 1.09 to 1.00 (PPI to PPII).

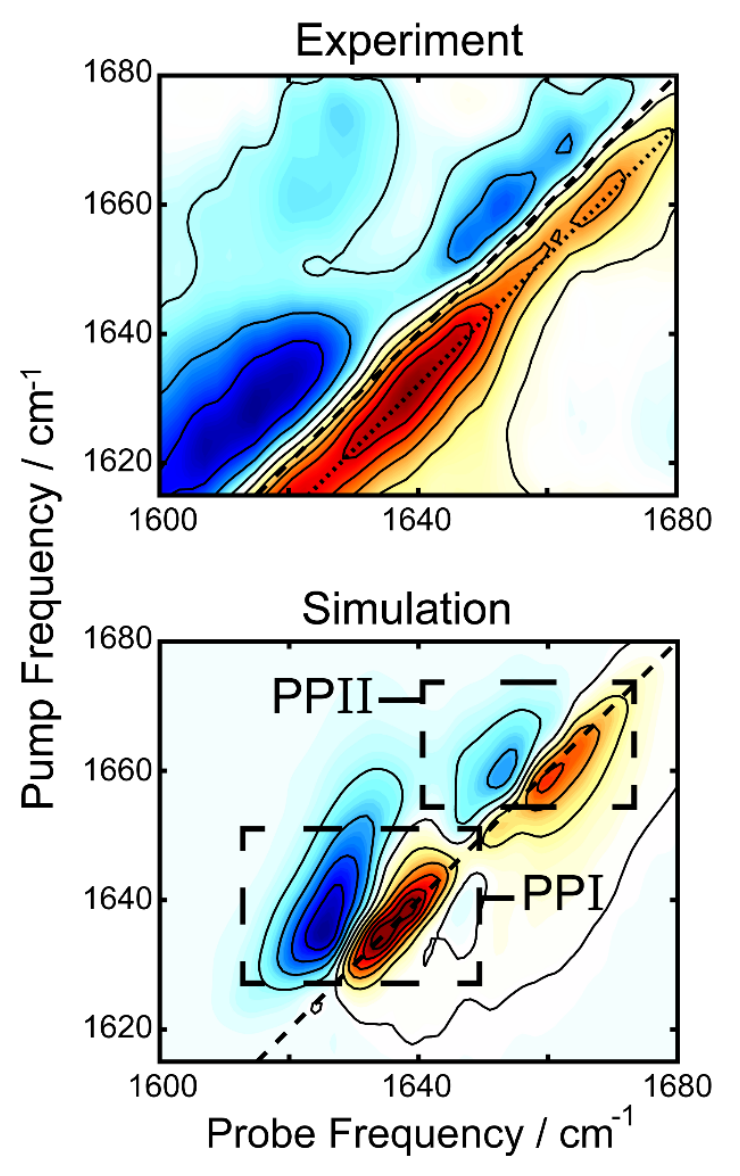


Figure 4. Comparison of the experimental difference 2DIR spectrum (top) and the sum of simulated 2DIR spectra for PPI and PPII helices (bottom), demonstrating the Amide I region of the former is well described by the latter.

Though previous evidence has shown that droplets can drive amyloid aggregation, ${ }^{41,42}$ these findings suggest that droplet formation can also promote single domain protein folding, which may serve as an early stage of the amyloid assembly process. While we believe that volumetric selfcrowding, whereby the high local concentration of $\mathrm{PR}_{20}$ peptide within droplets destabilizes extended structures, is likely the driving force for the folding of peptides into helices in the peptide dense phase, another contributing factor to stabilization may be inter-molecular pi stacking of the arginine sidechains, though any changes this may have induced it the guanidinium transitions in the 2DIR spectra are masked by the presence of the PEG signal near $1600 \mathrm{~cm}^{-1}$.

In conclusion, by performing FTIR, 2DIR and spectroscopic simulation on a model peptide, we have found, to our knowledge, the first evidence of LLPS driven peptide folding. These results could have great implications on the physical properties and functionality of liquid droplets.

METHODS:

\section{Peptide Synthesis and Purification}

The $\mathrm{PR}_{20}$ peptide was synthesized on a CEM Liberty Lite peptide synthesizer using conventional FMOC synthesis on a rink-amide resin. The peptide was cleaved using trifluoro-acetic acid (TFA) and precipitated with diethyl ether to retrieve the crude peptide. Crude peptide was purified using a Jasco LC-4000 HPLC system with a RP-C18 column. The HPLC fraction containing peptide was confirmed using MALDI-TOF mass spectrometry. The lyophilized peptide was resuspended in hexafluoroisopropanol with $10 \% \mathrm{v} / \mathrm{v} \mathrm{HCl}$ to exchange out TFA. The peptide was then lyophilized overnight and stored as a powder. To prepare samples for IR experiments, we 
resuspended our lyophilized peptide in deuterated hexafluoroisopropanol (HFIP-d) to exchange amide hydrogens with deuterium. The sample was incubated for at least 30 minutes and lyophilized to remove HFIP-d. This is repeated twice and, after the final step, the sample was lyophilized overnight to remove trace solvents.

\section{Sample Preparation}

To induce phase separation, we adapted a method described by Boeynaems and coworkers ${ }^{43}$. Lyophilized pure peptide was resuspended in $\mathrm{D}_{2} \mathrm{O}$ to a concentration of $1 \mathrm{mM}$. A $10 \times$ potassium phosphate buffer was made by combining $61.5 \mathrm{~mL}$ of $1 \mathrm{M} \mathrm{K}_{2} \mathrm{HPO}_{4}$ with $38.5 \mathrm{~mL}$ of $1 \mathrm{M} \mathrm{KH}_{2} \mathrm{PO}_{4}$.

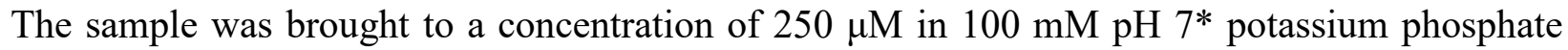
buffer with 30\% v/v PEG 300 (MW 300). The sample solution was placed on ice to induce droplet formation. Droplet formation was assessed by measuring turbidity at $600 \mathrm{~nm}$.

\section{Phase Contrast Microscopy}

A sample slide with a fluid reservoir formed by strips of double-sided tape was used to imaging droplets. The sample was placed in the reservoir and sealed with a coverslip. Images were taken using a Nikon TE2000-U microscope in phase contrast mode with a 40X objective.

\section{FTIR}

$10 \mu 1$ of sample solution was placed between two $2 \mathrm{~mm}$ thick $\mathrm{CaF}_{2}$ windows (Crystran Lmt.) with a Teflon spacer and tightly sealed to reduce atmospheric water exchange. Single beam spectra for sample and reference were collected using a Thermo Fischer Nicolet iS50R FTIR at $2 \mathrm{~cm}^{-1}$ resolution. Data was processed in MATLAB to produce the absorbance spectrum.

$2 D I R$

Details for the 2DIR laser system are described elsewhere. ${ }^{44}$ Briefly, $3.5 \mathrm{~mJ}, 30 \mathrm{fs}, 800 \mathrm{~nm}$ pulses from a $1 \mathrm{kHz}$ repetition rate Astrella Laser system (Coherent) are directed into a TOPAS prime 
OPA (Light Conversion) followed by a homebuilt $\mathrm{AgGaS}_{2}$ based $\mathrm{DFG}$ to produce our broadband mid-IR beam. The pump beam is sent through an AOM based mid-IR pulse shaper (PhaseTech Spectroscopy) in order to generate the pulse sequences necessary to perform 2DIR. The waiting time delay between the pump and probe pulses was set to $100 \mathrm{fs}$. The probe is directed through a sample and into a homebuilt monochromator where the spectrum is detected with a MCT focal plane array camera (Teledyne Catalina). The reference beam spectrum is detected simultaneously on the same camera. Data was processed using custom MATLAB code.

\section{ASSOCIATED CONTENT}

\section{Supporting Information}

2DIR spectrum of PEG 300 and details on simulation methods are included in the supporting information. Supporting Information is available free of charge on the ACS Publications website.

\section{ACKNOWLEDGMENT}

ALS gratefully acknowledges support from the College of Science at the University of Notre Dame. We thank Prof. Lin Guo of Jefferson University for helpful advice on droplet preparation protocols. We thank Prof. Anthony Serriani for access to the peptide synthesizer, and Prof. Holly Goodson for access to the Nikon TE2000-U microscope. DNE would like to thank the Notre Dame IBMS program and the Dana Wilson Quintero Memorial Graduate Fellowship for their support in this project. We would also like to thank Claire Nelmark for her help in assembling the manuscript.

\section{REFERENCES}

(1) Van Der Lee, R.; Buljan, M.; Lang, B.; Weatheritt, R. J.; Daughdrill, G. W.; Dunker, A. K.; Fuxreiter, M.; Gough, J.; Gsponer, J.; Jones, D. T.; et al. Classification of Intrinsically Disordered Regions and Proteins. Chem. Rev. 2014, 114 (13), 6589-6631. 
https://doi.org/10.1021/cr400525m.

(2) Linding, R.; Schymkowitz, J.; Rousseau, F.; Diella, F.; Serrano, L. A Comparative Study of the Relationship Between Protein Structure and $\beta$-Aggregation in Globular and Intrinsically Disordered Proteins. J. Mol. Biol. 2004, 342 (1), 345-353. https://doi.org/10.1016/J.JMB.2004.06.088.

(3) Pytowski, L.; Lee, C. F.; Foley, A. C.; Vaux, D. J.; Jean, L. Liquid-Liquid Phase Separation of Type II Diabetes-Associated IAPP Initiates Hydrogelation and Aggregation. Proc. Natl. Acad. Sci. 2020, 117 (22), 12050-12061. https://doi.org/10.1073/pnas.1916716117.

(4) Hyman, A. A.; Weber, C. A.; Jülicher, F. Liquid-Liquid Phase Separation in Biology. Annu. Rev. Cell Dev. Biol. 2014, 30 (1), 39-58. https://doi.org/10.1146/annurev-cellbio-100913013325.

(5) De Kruif, C. G.; Weinbreck, F.; De Vries, R. Complex Coacervation of Proteins and Anionic Polysaccharides. Curr. Opin. Colloid Interface Sci. 2004, 9 (5), 340-349. https://doi.org/10.1016/j.cocis.2004.09.006.

(6) Das, R. K.; Pappu, R. V. Conformations of Intrinsically Disordered Proteins Are Influenced by Linear Sequence Distributions of Oppositely Charged Residues. Proc. Natl. Acad. Sci. 2013, 110 (33), 13392-13397. https://doi.org/10.1073/pnas.1304749110.

(7) Danielsen, S. P. O.; McCarty, J.; Shea, J.-E.; Delaney, K. T.; Fredrickson, G. H. Molecular Design of Self-Coacervation Phenomena in Block Polyampholytes. Proc. Natl. Acad. Sci. 2019, 116 (17), 8224-8232. https://doi.org/10.1073/pnas.1900435116.

(8) Shin, Y.; Brangwynne, C. P. Liquid Phase Condensation in Cell Physiology and Disease. 
Science (80-. ). 2017, 357 (6357), eaaf4382. https://doi.org/10.1126/science.aaf4382.

(9) Brangwynne, C. P.; Tompa, P.; Pappu, R. V. Polymer Physics of Intracellular Phase Transitions. Nat. Phys. 2015, 11 (11), 899-904. https://doi.org/10.1038/nphys3532.

(10) Wang, Y.; Annunziata, O. Comparison between Protein-Polyethylene Glycol (PEG) Interactions and the Effect of PEG on Protein-Protein Interactions Using the Liquid-Liquid Phase Transition. J. Phys. Chem. B 2007, 111 (5), 1222-1230. https://doi.org/10.1021/jp065608u.

(11) Marianelli, A. M.; Miller, B. M.; Keating, C. D. Impact of Macromolecular Crowding on RNA/Spermine Complex Coacervation and Oligonucleotide Compartmentalization. Soft Matter 2018, 14 (3), 368-378. https://doi.org/10.1039/c7sm02146a.

(12) Courchaine, E. M.; Lu, A.; Neugebauer, K. M. Droplet Organelles? EMBO J. 2016, 35 (15), 1603-1612. https://doi.org/10.15252/embj.201593517.

(13) Patel, A.; Lee, H. O.; Jawerth, L.; Maharana, S.; Jahnel, M.; Hein, M. Y.; Stoynov, S.; Mahamid, J.; Saha, S.; Franzmann, T. M.; et al. A Liquid-to-Solid Phase Transition of the ALS Protein FUS Accelerated by Disease Mutation. Cell 2015, 162 (5), 1066-1077. https://doi.org/10.1016/j.cell.2015.07.047.

(14) Aumiller, W. M.; Keating, C. D. Phosphorylation-Mediated RNA/Peptide Complex Coacervation as a Model for Intracellular Liquid Organelles. Nat. Chem. 2016, 8 (2), 129137. https://doi.org/10.1038/nchem.2414.

(15) Ciryam, P.; Tartaglia, G. G.; Morimoto, R. I.; Dobson, C. M.; Vendruscolo, M. Widespread Aggregation and Neurodegenerative Diseases Are Associated with Supersaturated Proteins. 
Cell Rep. 2013, 5 (3), 781-790. https://doi.org/10.1016/j.celrep.2013.09.043.

(16) Cellmer, T.; Ferrone, F. A.; Eaton, W. A. Universality of Supersaturation in Protein-Fiber Formation. Nat. Struct. Mol. Biol. 2016, 23 (5), 459-461. https://doi.org/10.1038/nsmb.3197.

(17) Mukherjee, S.; Waegele, M. M.; Chowdhury, P.; Guo, L.; Gai, F. Effect of Macromolecular Crowding on Protein Folding Dynamics at the Secondary Structure Level. J. Mol. Biol. 2009, 393 (1), 227-236. https://doi.org/10.1016/j.jmb.2009.08.016.

(18) Brady, J. P.; Farber, P. J.; Sekhar, A.; Lin, Y.-H.; Huang, R.; Bah, A.; Nott, T. J.; Chan, H. S.; Baldwin, A. J.; Forman-Kay, J. D.; et al. Structural and Hydrodynamic Properties of an Intrinsically Disordered Region of a Germ Cell-Specific Protein on Phase Separation. Proc. Natl. Acad. Sci. 2017, 114 (39), E8194--E8203. https://doi.org/10.1073/pnas.1706197114.

(19) Johnson, W. C. Protein Secondary Structure and Circular Dichroism: A Practical Guide. Proteins Struct. Funct. Genet. 1990, $7 \quad$ (3), 205-214. https://doi.org/10.1002/prot.340070302.

(20) Shim, S.-H.; Zanni, M. T. How to Turn Your Pump-Probe Instrument into a Multidimensional Spectrometer: 2D IR and Vis Spectroscopies via Pulse Shaping. Phys. Chem. Chem. Phys. 2009, 11 (5), 748-761. https://doi.org/10.1039/b813817f.

(21) Bloem, R.; Garrett-Roe, S.; Strzalka, H.; Hamm, P.; Donaldson, P. Enhancing Signal Detection and Completely Eliminating Scattering Using Quasi-Phase-Cycling in 2D IR Experiments. Opt. Express 2010, 18 (26), 27067. https://doi.org/10.1364/OE.18.027067.

(22) Boeynaems, S.; Bogaert, E.; Kovacs, D.; Konijnenberg, A.; Timmerman, E.; Volkov, A.; 
Guharoy, M.; De Decker, M.; Jaspers, T.; Ryan, V. H.; et al. Phase Separation of C9orf72 Dipeptide Repeats Perturbs Stress Granule Dynamics. Mol. Cell 2017, 65 (6), 10441055.e5. https://doi.org/10.1016/j.molcel.2017.02.013.

(23) Wen, X.; Tan, W.; Westergard, T.; Krishnamurthy, K.; Markandaiah, S. S.; Shi, Y.; Lin, S.; Shneider, N. A.; Monaghan, J.; Pandey, U. B.; et al. Antisense Proline-Arginine RAN Dipeptides Linked to C9ORF72-ALS/FTD Form Toxic Nuclear Aggregates That Initiate In Vitro and In Vivo Neuronal Death. Neuron 2014, 84 (6), 1213-1225. https://doi.org/10.1016/j.neuron.2014.12.010.

(24) White, M. R.; Mitrea, D. M.; Zhang, P.; Stanley, C. B.; Cassidy, D. E.; Nourse, A.; Phillips, A. H.; Tolbert, M.; Taylor, J. P.; Kriwacki, R. W. C9orf72 Poly(PR) Dipeptide Repeats Disturb Biomolecular Phase Separation and Disrupt Nucleolar Function. Mol. Cell 2019, 74 (4), 713-728.e6. https://doi.org/10.1016/j.molcel.2019.03.019.

(25) Freibaum, B. D.; Lu, Y.; Lopez-Gonzalez, R.; Kim, N. C.; Almeida, S.; Lee, K. H.; Badders, N.; Valentine, M.; Miller, B. L.; Wong, P. C.; et al. GGGGCC Repeat Expansion in C9orf72 Compromises Nucleocytoplasmic Transport. Nature 2015, 525 (7567), 129-133. https://doi.org/10.1038/nature14974.

(26) Thompson, R. W.; Latypov, R. F.; Wang, Y.; Lomakin, A.; Meyer, J. A.; Vunnum, S.; Benedek, G. B. Evaluation of Effects of PH and Ionic Strength on Colloidal Stability of IgG Solutions by PEG-Induced Liquid-Liquid Phase Separation. J. Chem. Phys. 2016, 145 (18), 185101. https://doi.org/10.1063/1.4966708.

(27) Cohen, J. A.; Podgornik, R.; Hansen, P. L.; Parsegian, V. A. A Phenomenological OneParameter Equation of State for Osmotic Pressures of PEG and Other Neutral Flexible 
Polymers in Good Solvents. J. Phys. Chem. B 2009, 113 (12), 3709-3714. https://doi.org/10.1021/jp806893a.

(28) Vivarès, D.; Belloni, L.; Tardieu, A.; Bonneté, F. Catching the PEG-Induced Attractive Interaction between Proteins. Eur. Phys. J. E 2002, 9 (1), 15-25. https://doi.org/10.1140/epje/i2002-10047-7.

(29) Ghosh, A.; Tucker, M. J.; Hochstrasser, R. M. Identification of Arginine Residues in Peptides by 2D-IR Echo Spectroscopy. J. Phys. Chem. A 2011, 115 (34), 9731-9738. https://doi.org/10.1021/jp201794n.

(30) Barth, A.; Zscherp, C. What Vibrations Tell Us about Proteins. Q. Rev. Biophys. 2002, 35 (4), 369-430. https://doi.org/10.1017/S0033583502003815.

(31) Doyle, B. B.; Bendit, E. G.; Blout, E. R. Infrared Spectroscopy of Collagen and Collagenlike $\quad$ Polypeptides. $\quad$ Biopolymers $\quad \mathbf{1 9 7 5}, \quad 14 \quad$ (5), $\quad 937-957$. https://doi.org/10.1002/bip.1975.360140505.

(32) Dukor, R. K.; Keiderling, T. A. Mutarotation Studies of Poly-L-Proline Using FTIR, Electronic and Vibrational Circular Dichroism. Biospectroscopy 1998, 2 (2), 83-100. https://doi.org/10.1002/(SICI)1520-6343(1996)2:2<83::AID-BSPY2>3.0.CO;2-T.

(33) Fields, C. R.; Dicke, S. S.; Petti, M. K.; Zanni, M. T.; Lomont, J. P. A Different HIAPP Polymorph Is Observed in Human Serum Than in Aqueous Buffer: Demonstration of a New Method for Studying Amyloid Fibril Structure Using Infrared Spectroscopy. J. Phys. Chem. Lett. 2020, 6382-6388. https://doi.org/10.1021/acs.jpclett.0c01345.

(34) Hayashi, T.; Mukamel, S. Vibrational-Exciton Couplings for the Amide I, II, III, and A 
Modes of Peptides. J. Phys. Chem. B 2007, 111 (37), 11032-11046. https://doi.org/10.1021/jp070369b.

(35) Hamm, P.; Zanni, M. Concepts and Methods of 2D Infrared Spectroscopy; Cambridge University Press: Cambridge, 2011. https://doi.org/10.1017/CBO9780511675935.

(36) Demirdöven, N.; Cheatum, C. M.; Chung, H. S.; Khalil, M.; Knoester, J.; Tokmakoff, A. Two-Dimensional Infrared Spectroscopy of Antiparallel $\beta$-Sheet Secondary Structure. $J$. Am. Chem. Soc. 2004, 126 (25), 7981-7990. https://doi.org/10.1021/ja049811j.

(37) Adzhubei, A. A.; Sternberg, M. J. E.; Makarov, A. A. Polyproline-II Helix in Proteins: Structure and Function. J. Mol. Biol. 2013, 425 (12), 2100-2132. https://doi.org/10.1016/j.jmb.2013.03.018.

(38) Kang, Y. K.; Jhon, J. S.; Han, S. J. Conformational Study of Ac-Xaa-Pro-NHMe Dipeptides: Proline Puckering and Trans/Cis Imide Bond. J. Pept. Res. 1999, 53 (1), 3040. https://doi.org/10.1111/j.1399-3011.1999.tb01614.x.

(39) Ganim, Z.; Tokmakoff, A. Spectral Signatures of Heterogeneous Protein Ensembles Revealed by MD Simulations of 2DIR Spectra. Biophys. J. 2006, 91 (7), 2636-2646. https://doi.org/10.1529/biophysj.106.088070.

(40) Lessing, J.; Roy, S.; Reppert, M.; Baer, M.; Marx, D.; Jansen, T. L. C.; Knoester, J.; Tokmakoff, A. Identifying Residual Structure in Intrinsically Disordered Systems: A 2D IR Spectroscopic Study of the GVGXPGVG Peptide. J. Am. Chem. Soc. 2012, 134 (11), 50325035. https://doi.org/10.1021/ja2114135.

(41) Li, Y. R.; King, O. D.; Shorter, J.; Gitler, A. D. Stress Granules as Crucibles of ALS 
Pathogenesis. J. Cell Biol. 2013, 201 (3), 361-372. https://doi.org/10.1083/jcb.201302044.

(42) Wegmann, S.; Eftekharzadeh, B.; Tepper, K.; Zoltowska, K. M.; Bennett, R. E.; Dujardin, S.; Laskowski, P. R.; MacKenzie, D.; Kamath, T.; Commins, C.; et al. Tau Protein LiquidLiquid Phase Separation Can Initiate Tau Aggregation. EMBO J. 2018, 37 (7). https://doi.org/10.15252/embj.201798049.

(43) Boeynaems, S.; De Decker, M.; Tompa, P.; Van Den Bosch, L. Arginine-Rich Peptides Can Actively Mediate Liquid-Liquid Phase Separation. Bio-Protocol 2017, 7 (17). https://doi.org/10.21769/bioprotoc.2525.

(44) Cracchiolo, O. M.; Geremia, D. K.; Corcelli, S. A.; Serrano, A. L. Hydrogen Bond Exchange and $\mathrm{Ca} 2+$ Binding of Aqueous $\mathrm{N}$-Methylacetamide Revealed by 2DIR Spectroscopy. J. Phys. Chem. B 2020, acs.jpcb.0c02444. https://doi.org/10.1021/acs.jpcb.0c02444. 
TOC GRAPHICS

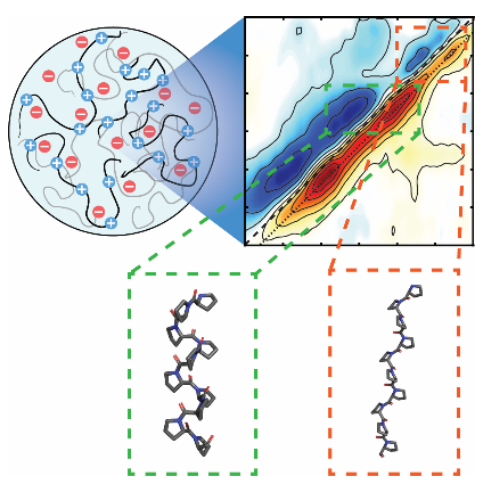




\title{
Supporting Information for "Does Liquid-Liquid Phase Separation Drive Peptide Folding?"
}

\author{
Dean N. Edun, Meredith R. Flanagan, Arnaldo L. Serrano* \\ Department of Chemistry and Biochemistry, University of Notre Dame, Notre Dame, \\ Indiana 46556, United States \\ Corresponding Author \\ *arnaldo.serrano@nd.edu
}




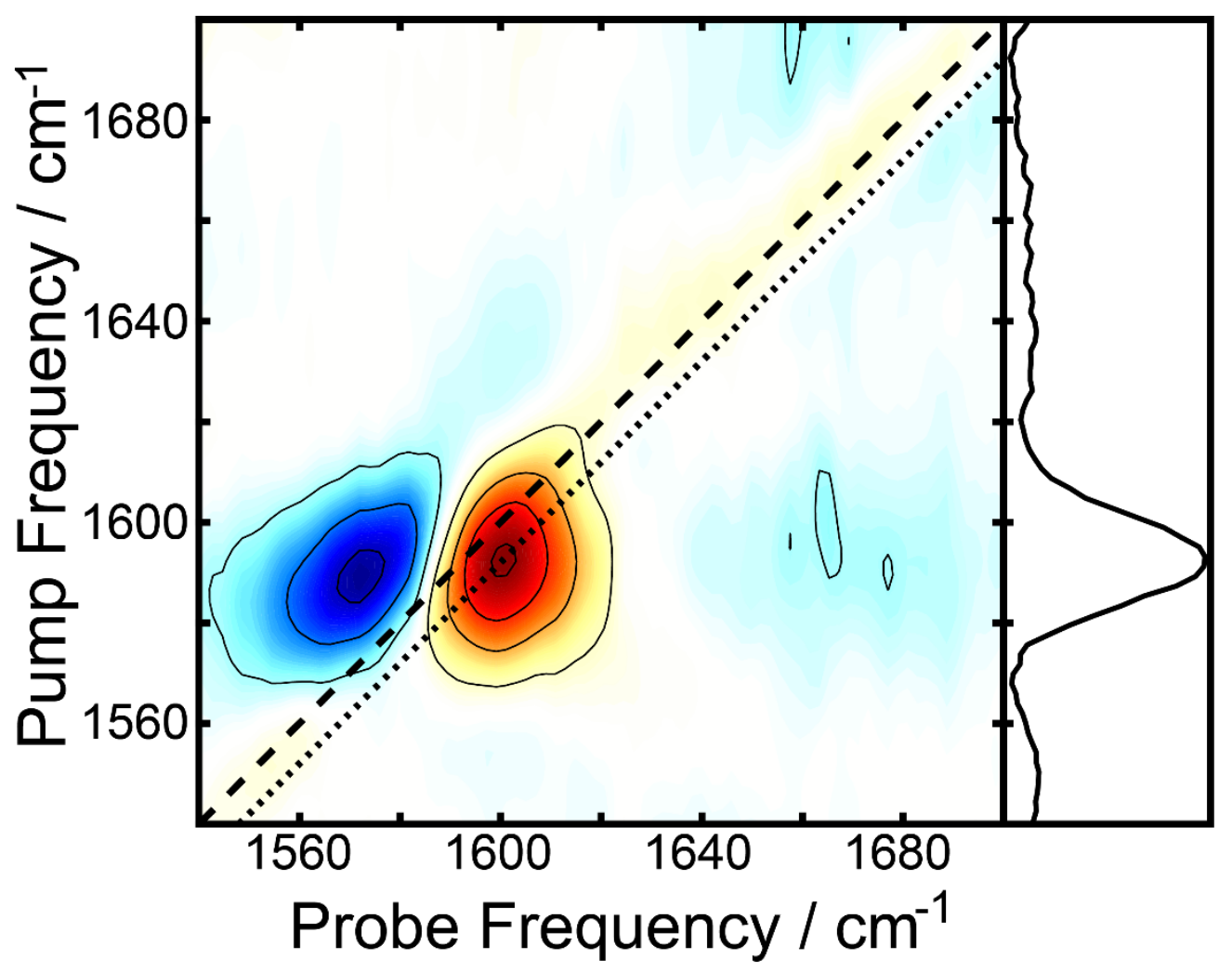

Figure S1. 2DIR spectrum of PEG in $\mathrm{D}_{2} \mathrm{O}$. We attribute the peak that arises at around $1600 \mathrm{~cm}^{-1}$ pump frequency in other spectra to PEG. 
Methods for simulating 2DIR spectra

The spectra were simulated using a floating oscillator model where we extract the amide-I transition dipole moment positions and directions from idealized PPI and PPII structures and calculate the transition dipole coupling energy between all pairs of dipoles. The amide-I central frequencies were set to $1650 \mathrm{~cm}^{-1}$. Gaussian diagonal disorder to the exciton Hamiltonian of 10 $\mathrm{cm}^{-1}$ standard deviation was included. Diagonalization of the coupling energies provides normal mode frequencies and energies, from which 2DIR stick spectra can be generated. Stick spectra were averaged over 800 realizations of diagonal disorder. The final simulated 2DIR spectra were obtained by convolving the 2D stick spectrum with 2D Lorentzian line-shape functions with $3 \mathrm{~cm}^{-1}$ half-width at half max. ${ }^{1}$ 


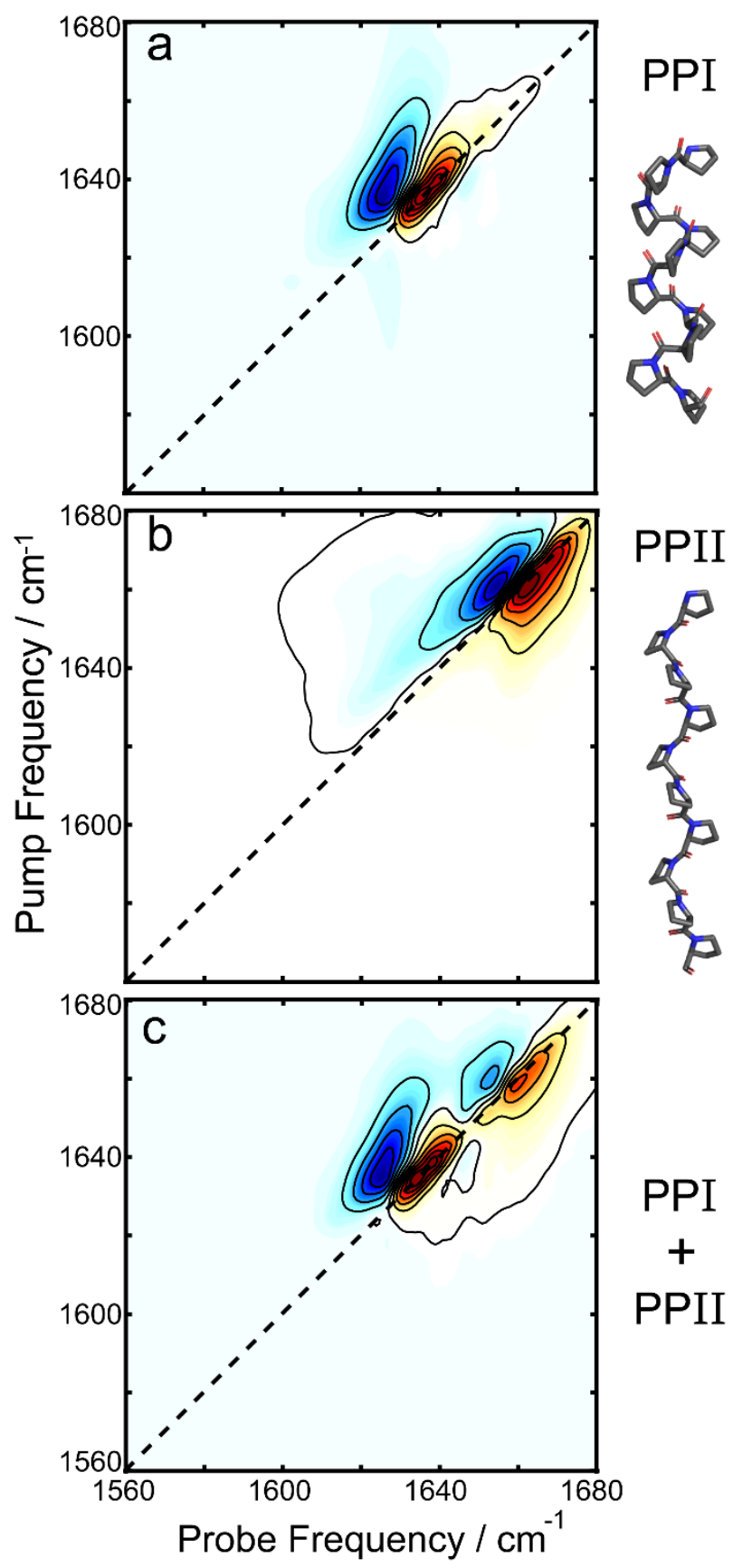

Figure S2. a) Simulated 2DIR spectrum of PPI structure. b) Simulated 2DIR spectrum of PPII structure. c) Summed spectra of both polyproline structures. The associated structure is shown on the right for each spectrum. 
(1) Ganim, Z.; Tokmakoff, A. Spectral Signatures of Heterogeneous Protein Ensembles Revealed by MD Simulations of 2DIR Spectra. Biophys. J. 2006, 91 (7), 2636-2646. https://doi.org/10.1529/biophysj.106.088070. 